\title{
Innovación en emprendimientos periodísticos y de comunicación frente a las nuevas tendencias digitales
}

\author{
Innovation in journalistic and communication ventures \\ in the front of the new digital trends.
}

(D) Ana Paulina Escobar

Ana-Paulina_Escobar@etu.u-bourgogne.fr

Universidad de Borgoña (Francia)

https://orcid.org/0000-0003-4921-8839
(D) Liza Bahamonde

libahamondezu@uide.edu.ec

Universidad Internacional del Ecuador (Quito)

https://orcid.org/0000-0003-0306-0722

DOI https://doi.org/10.32719/26312514.2020.3.6

\section{Resumen}

En el mundo, los emprendimientos de periodismo y de comunicación se han ido innovando frente a la era digital. También conocidos como startups, revelan en general la voluntad por iniciar un proyecto, por innovar, por renovar. En este artículo, reflexionamos sobre los conceptos que describen el escenario de los emprendimientos, como la apropiación digital y las mutaciones de las dinámicas de trabajo; así también, exploramos la realidad de dos emprendimientos ecuatorianos: el portal de periodismo narrativo La Barra Espaciadora y la agencia de comunicación Orion. Al estudiarlos, hemos constatado características comunes así como particularidades propias al tipo de emprendimiento, que en conjunto revelan los cambios que han venido experimentando la comunicación y el periodismo en la era digital.

\begin{abstract}
In the world, journalism and communication entrepreneurships have been innovating in the face of digital era. Also known as startups, these entrepreneurships reveal in general the wish to start a project, to innovate, to renew. In this article, we reflect one of the concepts that describe the entrepreneurships scenario, such as digital appropriation and mutations of work dynamics; likewise, we explore the experience of two projects: the portal of narrative journalism La Barra Espaciadora and the communication agency Orion. When studying them, we have observed common characteristics as well as specific particularities, which together reveal changes in both journalism and communication in the digital era.
\end{abstract}

\section{Palabras clave $\cdot$ Keywords}

Emprendimientos digitales; periodismo innovador; comunicación; innovación. Digital entrepreneurships; innovating journalism; communication; innovation. 


\section{Introducción}

El talento y la necesidad son factores que se unen a la hora de emprender. Los profesionales que lo hacen han encontrado en la innovación las herramientas para darle un giro a su actividad laboral o para reemplazarla por una nueva. El perfil de startups españolas evidenció ya en 2015 que la mayoría de proyectos son personales, motivados por la inquietud de una idea innovadora y se especializan en servicios digitales más que en nuevos productos; $y$ en sus tres primeros años transforman la vida del emprendedor (Top Seeds Lab 2015).

En el campo de la comunicación, el profesional se enfrenta a la apropiación de recursos tecnológicos, considerando los retos que impone la cultura digital (Fernández, Vallejo y McAnally 2015). Nuevas dinámicas de trabajo al servicio de la comunicación, del cambio social y de la democracia son factores que marcan el día a día de un comunicador, un periodista, un consultor para organizaciones e instituciones como un fenómeno que no es ajeno a Ecuador.

En muchos casos, los emprendimientos pueden evidenciar una mutación en la carrera profesional, mientras que, en otros casos, su continuidad, pero con un giro innovador y en función de valores éticos con los que los profesionales se identifican. En ambos casos, los emprendimientos se dejan guiar por las potencialidades de la era digital (Quezada y Pérez 2016), por la posibilidad de experimentar cambios en las rutinas de trabajo, así como de - en medio de esta rutina-incorporar nuevos recursos. A la vez, es importante considerar en este contexto los conceptos de hipermediación, como un proceso que interconecta a "sujetos, medios y lenguajes" y el de la interfaz, como una herramienta que facilita la comunicación; en conjunto, como conceptos clave del entorno en el que se desenvuelven los comunicadores contemporáneos.

En nuestro estudio, nos proponemos analizar el uso de recursos, aplicaciones y plataformas digitales por parte de profesionales emprendedores así como la incorporación de nuevas prácticas de trabajo, como una evidencia de su disposición por innovar, por adaptarse a nuevas dinámicas de trabajo o a nuevas trayectorias profesionales; sin embargo, no desde la perspectiva del determinismo tecnológico, sino colocando a las personas como protagonistas de estos cambios.

Por ello, nuestro objetivo es identificar las nuevas prácticas y estrategias, así como los recursos tecnológicos que utilizan los emprendimientos de periodismo y comunicación, particularmente La Barra Espaciadora y Orión, para establecer cómo se han ido innovando frente a las tendencias de la era digital.

\section{Metodología}

Para nuestro análisis, hemos escogido dos emprendimientos digitales comunicacionales: uno periodístico y uno de comunicación. En el primer caso, analizamos al portal 
de periodismo narrativo La Barra Espaciadora; y, en el segundo caso, a la agencia de comunicación Orión.

Nuestra investigación utiliza una metodología cualitativa, un método que nos permitió comprender la realidad de cada emprendimiento, específicamente en el campo del periodismo y de la comunicación. Para ello, realizamos entrevistas de profundidad con los representantes de cada emprendimiento, tomando a este instrumento como una técnica válida y complementaria a la investigación cualitativa (Coffey y Atkinson 2003); además de una breve observación de las páginas web de estos emprendimientos.

Previamente, habíamos considerado como hipótesis el hecho de que las prácticas profesionales respecto a innovación son similares tanto en los emprendimientos de periodismo como en los de comunicación y que, por tanto, los cambios en las dinámicas de trabajo eran guiados por la apropiación tecnológica y la inclusión de estas prácticas. Sin embargo, el análisis de las entrevistas sobre la base de los conceptos teóricos nos permitió identificar características particulares en cada emprendimiento.

\section{Marco teórico}

\subsection{Apropiación y cultura tecnológica}

La disponibilidad y el uso de recursos tecnológicos en la rutina de trabajo son aspectos que caracterizan la cotidianidad laboral de diferentes grupos profesionales. ¿Se trata de nuevas dinámicas de trabajo o de la incorporación de tareas y actividades propias de la era digital? En el campo de la comunicación, los diversos recursos, dispositivos, aplicaciones y plataformas digitales han cambiado o, por lo menos, han facilitado las rutinas laborales de profesionales como el periodista, el comunicador, el consultor de comunicación para empresas, organizaciones e instituciones. Aquí, surgen nuevas interrogantes: ¿se trata de prácticas y usos que los comunicadores incorporan en su rutina laboral? o ¿de estrategias que aplican en función de la apropiación que hacen los usuarios de ciertos recursos, como las redes sociales?

En el contexto de las redes sociales y tomando como base la teoría de las relaciones de Ledingham y Bruning, por ejemplo, los investigadores Charest, Gauthier y Grenon establecen 10 pasos que los profesionales de la comunicación deben tomar en cuenta para incorporar un proceso estratégico de apropiación e integración de redes en sus estrategias de comunicación (Charest, Gauthier y Grenon 2013). Nosotros agrupamos estos pasos en tres etapas: la inicial: 1) observación del entorno de las redes, 2) análisis de las necesidades internas y externas, 3 ) identificación de los públicos o clientes además de sus objetivos; la de implementación: 4) elección de la herramienta adecuada y creación de perfiles, 5) planificación de contenidos para cada medio o red y normas de uso, 6) elaboración de herramientas de gestión, 7) definición del administrador o administradores de la comuni- 
dad virtual; y la de evaluación: 8) generar interactividad, 9) crear comunidades de interés además de 10) evaluar todos los pasos de la estrategia.

Es claro que la incorporación de estos pasos dependerá del tipo de organización, así como de la capacidad de cada comunicador para identificar las tendencias y los niveles de apropiación de los internautas o usuarios de las redes. En cuanto al comunicador, la asimilación de estas consideraciones facilitará su proceso de apropiación, así como la integración de cambios en su rutina de trabajo.

En nuestro análisis, es relevante destacar el rol del profesional como protagonista de este cambio y no necesariamente el que se le atribuye a la tecnología. Hay estudios que han explorado el componente tecnológico, que implica diferentes elementos y una diversidad de actores. Por ejemplo, el proceso de apropiación tecnológica de la telegrafía sin hilos a la radiofonía, tal como la conocemos hoy en día (Quezada y Pérez 2016), evidencia la evolución de la radio como un proceso que ha involucrado a aficionados, inversionistas, colaboradores de la prensa, entre los principales actores; pero, no solo eso, sino sobre todo, la idea de que no son "los artefactos" los que determinan la historia tecnológica, sino nuestras decisiones sobre esos artefactos.

En el campo del periodismo, otro aporte analiza la capacidad de apropiación tecnológica por parte de periodistas ecuatorianos, en el uso de redes sociales (Escobar 2018). Al igual que en el caso de la radio, no se trata de una adaptación de los periodistas a los cambios, en función de lo que imponen las redes sociales, sino de una incorporación y uso consciente de redes sociales, como Twitter, en sus dinámicas de trabajo, como producción y difusión de la información.

La importancia de conocer el concepto apropiación tecnológica radica justamente en que nos permite reflexionar sobre el uso consciente de la tecnología en la sociedad, en general; pero no solo en ello, sino en que permite mejorar las condiciones de los sistemas educativos a través de canales efectivos de distribución del conocimiento, que desde nuestra perspectiva se trata más bien de una "gestión, mediación y comunicación del conocimiento", más que una mera distribución.

En el contexto educativo, con el tiempo, este proceso inicial de asimilación del conocimiento y de la tecnología que empieza en las aulas otorgará más herramientas a los futuros profesionales tanto en su vida cotidiana como en su desempeño laboral (Fernández, Vallejo y McAnally, 2015). Asimismo, considerando el énfasis que hacen los autores precedentes en torno a la tradición psicosocial para entender el concepto de apropiación tecnológica, este proceso también multiplica la capacidad de decisión del consumidor frente a la oferta del mercado, al adquirir y utilizar servicios y dispositivos; así también, la inclusión de estrategias para mejorar la adaptación tecnológica en las organizaciones y la capacidad de la gestión pública digital, en los sistemas de gobierno. 


\subsection{Mutación y transformaciones sociales}

Cuando hablamos de mutación, estamos hablando de cambio, de transformaciones, en general. Estas transformaciones no se limitan a los efectos derivados de los desarrollos y usos tecnológicos; tienen que ver también y sobre todo con el cambio de la sociedad que habitamos y de la que formamos parte.

Entonces, ¿cuáles son las transformaciones que ocurren en el campo de la comunicación? y - como sociedad- ¿ cómo estamos cambiando? Somos una sociedad en plena mutación y en la que todo está cambiando, desde las instituciones hasta la misma familia, como tradicional célula de la sociedad (Fundación Friedrich-Ebert-Stiftung 2015). Han cambiado las nociones de progreso, de la información y hasta el propio modelo tradicional de los medios de comunicación, nociones que, en conjunto, evidencian lo que para Jesús Martín Barbero toma el nombre de "las hablas".

La información es un motor, el motor mismo de esta sociedad en mutación porque es la que ha permitido la configuración de nuevos tipos de información como la información social, que resume los modos en que - a través de datos- nos comunicamos tanto con la escuela y el hospital, como con los bancos y las instituciones de control; e incluso la información genética, que ha cambiado el mundo de la ciencia (Fundación Friedrich-Ebert-Stiftung 2015, 15). Es la que incluso marca una diferencia entre la sociedad donde imperaba la ley del más fuerte y aquella, en la que hoy vivimos, donde predomina la ley del más sabio, aunque, desde nuestra perspectiva, es más pertinente hablar de la ley del que más información posee.

$\mathrm{Al}$ referir estos cambios, estamos hablando de cómo han cambiado nuestros hábitos, nuestras prácticas profesionales y nuestras formas de relacionarnos, lo cual implica que, para entender estas transformaciones, es necesario fijarse en la sociedad y sus cambios más que en la tecnología.

En el caso particular del periodismo, Mercier y Pignard-Cheynel (2014) destacan la importancia de las transformaciones profundas, de "mutaciones" tanto en las redacciones, con el uso de nuevas tecnologías, así como en la adquisición de nuevas competencias, por parte de los periodistas. Su trabajo analiza las características y los desafíos del periodismo digital en Francia, destacando un aporte propio, pero también el trabajo académico precedente publicado en revistas francófonas.

En el caso de las estructuras organizacionales de las redacciones, desde la perspectiva de la sociología del periodismo, se evalúa a la profesión, en función de las nuevas competencias que exige el oficio, pero también considerando la redefinición de la identidad profesional. Y, en cuanto al rol del periodista, los autores ponen en evidencia los estudios sobre usos y apropiación de innovaciones que han permitido asimilar la forma en que los periodistas han adoptado o no las nuevas herramientas tecnológicas, experimentando transformaciones al utilizar la tecnología o, en otros casos, intentando "dominarla". 
También pasa por su análisis el impacto de la producción periodística y no solo se detiene en los estándares que supone el oficio, sino que considera además que en esta nueva producción han surgido oficios como el de los infografistas, diseñadores web, desarrolladores de contenidos, que también otorgan características a la mutación que ha experimentado el periodismo en la era digital.

Aunque no entra en el análisis de Mercier y Pignard-Cheynel, el campo de la comunicación no queda exento de estas transformaciones. Los comunicadores son también gestores de contenidos multimedia, administradores de comunidades virtuales y al mismo tiempo desempeñan la actividad esencial y tradicional que es la gestión de la comunicación para organizaciones, instituciones o empresas; es decir, han aprendido a desempeñar diversas actividades nuevas a la par de las tradicionales, incorporándolas simultáneamente dentro de su rutina de trabajo, como ocurre en el caso de la incorporación de redes sociales en sus estrategias de comunicación.

\subsection{Emprendimientos y nuevas dinámicas de trabajo en la era digital}

Los emprendimientos no son un efecto de la era digital. Siempre han existido. Lo que ha hecho la era digital es facilitar la visibilización de iniciativas profesionales que han saltado de los formatos convencionales a las plataformas digitales.

El emprendimiento implica a la vez la puesta en marcha de iniciativas, como una obra, un negocio o empeño (Real Academia Española [RAE] 2019a), pero también la descripción de los emprendedores como personas con pensamiento creativo e innovador, con habilidades gerenciales y con vocación de buscar logros y enfrentar riesgos (Corporación Andina de Fomento 2013). Muchas de estas iniciativas económicas surgen de necesidades o de oportunidades que se presentan en el mercado.

En 2017, Ecuador, como parte del proyecto de investigación Global Entrepreneurship (GEM), la Escuela de Negocios de la Espol elaboró una encuesta de emprendimiento en con el fin de medir el nivel de actividad emprendedora, analizar los factores que determinan estas actividades e identificar las oportunidades para diseñar políticas públicas y recomendaciones para mejorar el nivel de actividad emprendedora. Replicando el modelo iniciado por Babson College y London Business School, el GEM realizó una radiografía sobre el emprendimiento en 54 países de los cinco continentes, además de un perfil del emprendedor (Espae-Espol 2018).

En el caso de Ecuador, el emprendedor es una persona con 36 años de edad, en promedio, un negocio orientado al consumidor y 11 años de escolaridad. Además, 48,8 \% de los encuestados (2097 adultos, de los cuales 37 son expertos nacionales) provienen de hogares con ingresos mensuales entre 375 y 750 dólares, mientras que el 33,4 \% tiene un empleo adicional.

Los resultados demostraron también que, a 2017, alrededor de tres millones de 
ecuatorianos emprendieron un negocio o tenían uno con menos de cuatro años, lo que representó al 29,6 \% de la población entre 18 y 64 años de edad, siendo la tasa de actividad emprendedora temprana (TEA) más alta de la región. Un hallazgo preocupante en este reporte, sin embargo, fue que la mayoría de emprendimientos no genera empleo, lo cual ocurre en el $99 \%$ de los negocios nacientes, el $60 \%$ de los negocios nuevos y en el 51,5\% de los negocios establecidos.

Por otro lado, una edición de Open Society Foundations y el Centro Knight para el Periodismo en las Américas dio cuenta en 2017 de un conjunto de proyectos de periodismo innovador en América Latina, que revelan principalmente la incorporación progresiva de recursos tecnológicos y prácticas renovadoras en proyectos y emprendimientos de periodismo (Centro Knight para Periodismo en las Américas 2018). En primer lugar, se destaca el uso de plataformas como Periscope y Facebook en noticieros de televisión y portales digitales, además de mapas interactivos y realidad virtual, entre otros recursos audiovisuales, que permiten generar interactividad con las audiencias; y, en segundo lugar, se destacan proyectos que buscan renovar la calidad de los enfoques, tanto en investigación como en información, dirigida a audiencias específicas como la de los denominados "millennials"; $y$, en esta misma línea sobresalen emprendimientos especializados en fact checking y periodismo de datos que, en conjunto, reconfiguran las nuevas prácticas del periodismo en la era digital.

Por otra parte, el informe también evidencia la apuesta del emprendedor, por buscar simultáneamente logros y enfrentar riesgos. Por ejemplo, sobresale el desarrollo de una startup creada por un periodista en Brasil, tras ser despedido y que en lo posterior generaría trabajo para 20 periodistas y, también, la definición de una creativa estrategia publicitaria por parte de un proyecto venezolano para asegurar rentabilidad; esto, a través de una fórmula que ofrece a los anunciantes la posibilidad de aparecer en tres portales periodísticos por el precio de uno.

Estos ejemplos no solo muestran la capacidad emprendedora e innovadora en proyectos periodísticos, sino también que sus ejecutores han aprovechado las oportunidades de la era digital para lograr su permanencia en el mercado laboral y, a la vez, facilitar el ingreso de nuevos profesionales.

En Ecuador, los emprendimientos periodísticos, pioneros del espacio digital fueron Ecuador Inmediato y Ciudadanía Informada que, en 2004, marcaron el camino para muchos proyectos más.

\subsection{Hipermediación e interfaz, claves para entender el entorno del comunicador}

Las tecnologías no solo transforman al mundo, sino que también influyen en la percepción que los sujetos tienen de ese mundo (Scolari 2013). Para Scolari, la llegada a nuestro 
hogar de tecnologías como la radio, la televisión y el internet creó límites que separan lo interior de lo exterior, pero estos límites al mismo tiempo son permeables y permiten un intercambio. A ello podríamos añadir que la comunicación, entendida como un organismo abierto genera información al interior del mismo y a la vez esta información puede influir en el exterior o viceversa. Un ejemplo es lo que sucede en las redes sociales que actualmente contienen la información personal que generamos y que en algunos casos puede tener un impacto macro en nuestro entorno. Así también, en la declaración de nuestras posturas sociales o políticas, estas pueden generar diversas reacciones en nuestras actividades cotidianas.

En sus inicios, el internet sirvió para respaldar la idea de que la cooperación y la libertad de información pueden favorecer la innovación en mayor medida que la competencia y los derechos de propiedad. Además, sirvió para comprender su evolución posterior. Internet nació en la insólita encrucijada entre la gran ciencia, la investigación militar y la cultura libertaria. Los estudios "cibernéticos" inaugurados por Norbert Wiener en su célebre Cybernetics, or Control and Communication in the Animal and Machina, de 1948, establecen un primer acercamiento académico al término.

El estudio de la cibernética (estrechamente relacionada con los programas militares de la Guerra Fría, especialmente en el campo de la balística) (Gómez Cruz 2007), buscaba introducir elementos reflexivos como la información, la retroalimentación y la regulación no solo en sistema de ingeniería, sino en organismos vivos e incluso en el mismo lenguaje.

Pero, ¿la presencia de las interfaces es algo propio de la era digital? No, las interfaces siempre estuvieron ahí, desde mucho antes de la invasión de las pantallas interactivas. Nacieron cuando el Homo habilis recogió una piedra y la convirtió en un arma para cazar o defenderse. Por eso, cuando hablamos de interfaz, aunque nos parece un término lejano a nuestra realidad, debemos comprender que es algo que ha estado como parte de nuestra evolución, que enfrentamos con diferentes tipos de interfaces cada momento de nuestra vida diaria y es tan simple como apretar el interruptor de la luz para que esta se encienda.

Para algunos teóricos, la mejor interfaz es la que no necesita instrucciones: si la interfaz ha sido bien diseñada, las instrucciones se encuentran inscritas en su superficie y pueden ser fácilmente interpretadas por el usuario.

\subsection{Emprendimiento y recursos en línea}

Podemos afirmar que un nuevo mapa del mundo de la comunicación se está configurando. Sus rasgos característicos son la emergencia de la diversidad y la integración democrática en los diferentes tipos de organizaciones que definen el sistema social, el cual se constituye a través de la comunicación. La primera definición de sociedad para la RAE (2019b) se refiere al conjunto de personas, pueblos o naciones que conviven bajo normas comunes. Sin embargo, lo que tradicionalmente llamamos "sociedad", el conjunto de las ciencias sociales, la comunicación y la información lo han convertido en sistema social, 
a partir de la cibernética. La sociedad no es la suma de las personas que viven en un territorio determinado, sino que se define por los contactos, las relaciones, la comunicación y el intercambio de información, que es, todo ello en conjunto, el pegamento que constituye la sociedad como sistema (Costa 2015).

Para la comunicación, el emprendimiento tiene algunas particularidades, entre ellas el debilitamiento de las barreras de entrada tradicionales - tecnología y regulación-, que explican el número creciente de medios que tratan de conquistar el tiempo del público, su dinero y los presupuestos publicitarios de los anunciantes (Pérez-Latre y Sánchez-Tabernero 2012). Las ventajas competitivas de toda empresa de comunicación suelen provenir de factores intangibles como la gestión de talento y creatividad.

Existen diferentes tipos de redes sociales según su uso: la principal, por ser la más conocida, es Facebook, creada para fomentar la unión estudiantil en una universidad de Estados Unidos y que, en su alcance, ha evolucionado desde ser un medio de contacto con amigos y familiares hasta volcarse a las empresas y negocios; en este último aspecto, ha extendido sus servicios a la creación de tiendas virtuales y una diversidad de servicios (Gómez Cruz 2007).

Dentro de los nuevos actores sociales, el nuevo mapa del mundo de la comunicación está dominado por dos ideologías hegemónicas: la vía de discurso único de la economía y el impacto de las tecnologías con el desasosiego causado por las redes sociales como algo imposible de controlar (Costa 2015).

Como en algunos casos suele existir una relación directa entre la edad, la educación superior y el uso de internet, aquellos usuarios que dominan las herramientas y recursos tecnológicos, pueden utilizar el potencial comunicador de internet para crear y expandir un emprendimiento o una nueva forma de trabajo, como lo que está sucediendo en la actualidad. En el caso de la comunicación, ha sido el punto de partida o el impulso para el desarrollo de emprendimientos profesionales.

En este contexto, cabe indicar que existe cierta afinidad entre los procesos de emprendimiento y comunicación. El esquema "empresa-producto-consumidor" es similar al clásico modelo "emisor-mensaje-receptor". El emprendedor es alguien que crea y distribuye valor entre los consumidores específicos y obtiene un beneficio Un comunicador es alguien que crea y distribuye contenido entre públicos específicos y obtiene a cambio alguna forma de respuesta o retroalimentación. Quizá la capacidad del comunicador de ponerse en el lugar de sus públicos le facilite la detección de necesidades insatisfechas que inspiren ideas de negocio (Montalvo- Castro 2016).

Los públicos de la organización son quienes dan sentido final a su existencia, ya que son ellos con quienes la empresa busca entrar en contacto comercial, público y societario. La imagen de la empresa se forma en ellos gracias a ellos. Mientras más reducida sea la categoría de públicos a los que estemos estudiando, mayores serán las posibilidades de reconocer sus necesidades desde una perspectiva microsicológica (Garrido 2004). 
Así también, mientras más personal sea la experiencia que encontramos en los colectivos de públicos y grupos, más probable es la permanencia de la imagen generada conforme a los significados asociados e interpretados por el individuo en su experiencia, que pueden ser reforzados por el grupo en lo interpersonal o a través de información entregada por los medios de comunicación masivos. Al mismo tiempo, la experiencia personal será más valiosa para el público a la hora de configurar la imagen de una empresa; es lo que se ha demostrado aún en el caso de que la información entre dos canales sea similar (Gutiérrez y LePorte 2013).

Sabemos que, para la comunicación en las organizaciones, es crucial el trabajo con sus diferentes públicos; por ello, es también importante saber articular un mensaje efectivo, según los públicos de interés, conocidos como stakeholders. Cuando, en comunicación, señalamos que aspiramos a cambiar actitudes, modos de pensar y comportamientos de nuestro grupo objetivo estamos explicitando nuestro interés en que ese "alguien" y esos "algunos" se comporten en los rangos que esperamos y en favor de los fines económicos y sociales de la compañía. Ahí la importancia de que el comunicador identifique a cada uno de sus públicos para mejorar la información con la que va a diseñar sus estrategias de comunicación (Garrido 2004).

\section{Resultados}

Aun cuando los proyectos se desarrollan en un contexto digital, no todos innovan. El interés por innovar depende de las características de cada emprendimiento, del tipo de emprendimiento y, sobre todo, de cada emprendedor. No es desconocimiento de las potencialidades de la tecnología en favor de la innovación, sino una postura consciente de los profesionales con respecto a la utilidad de la tecnología y de las herramientas digitales, una postura que también describe las características y diferencias entre el mundo del periodismo y el mundo de la comunicación.

En el caso del periodismo, cuando hablamos de innovación en las prácticas profesionales, si estamos hablando del uso de la tecnología, de dispositivos y de herramientas que no solo ocurre en los medios tradicionales (Rivera y Rosero 2017), sino también desde la perspectiva de nuevos emprendimientos digitales o proyectos periodísticos puntuales (Knight Center Journalism in the Americas 2017). Es decir, el desarrollo de emprendimientos y la cotidianidad de la profesión en el campo del periodismo y la comunicación describen una constante innovación.

\subsection{Innovación en La Barra Espaciadora (LBE)}

La Barra Espaciadora es una revista digital de periodismo narrativo que fue fundada en 2013 por un grupo de periodistas que vio en internet una oportunidad para desa- 
rrollar una propuesta y una apuesta editorial independiente, con relación a lo que, en la época, ofrecía el periodismo tradicional. La intención no solo fue dar un salto desde los formatos que muchos de ellos aprendieron en el periodismo escrito al periodismo digital, sino también multiplicar la posibilidad de integrar a nuevas audiencias.

Así, además de presentarse como un medio especializado en contenidos de periodismo narrativo, buscó, desde el principio, incorporar entre sus colaboradores a periodistas y profesionales de distintas áreas que aportaran con crónicas y reportajes sobre los temas que no tenían y, sobre todo, no encontraban espacio en los medios tradicionales; o que, si lo encontraban, era en la agenda coyuntural (la noticia). Por ejemplo, los temas de derechos humanos y medio ambiente.

En este ejercicio de multiplicar voces, LBE también promueve una oferta de formación, a través de una escuela de periodismo que ofrece "talleres y clínicas periódicas", dirigidas tanto a estudiantes universitarios como a profesionales que deseen unirse con la perspectiva de colaborar con contenidos.

Estas dos iniciativas (involucrar nuevas voces a través de las fuentes y a través de los colaboradores) han sido facilitadas por los espacios promocionales que se ofrecen de manera permanente en la página web del proyecto periodístico. Banners laterales (en la parte derecha de la página) informan constantemente sobre estos servicios y son la evidencia de un buen uso de los recursos tecnológicos, pero también del rol de los periodistas como protagonistas de este proceso de esta adaptación tecnológica, de apropiación (Quezada y Pérez 2016).

En la página web de LBE, en su portada o sección "Inicio", se promociona los trabajos más leídos, en la etiqueta de 'Más populares ahora'; otros destacados en 'Visor', 'Mundo, 'Entre Nos', 'Nuestros últimos artículos', además de un enlace para el canal de YouTube de LBE y dos adicionales para dos medios 'Medios Amigos', que son el portal ecuatoriano de periodismo 'La andariega' y la red de medios latinoamericanos 'Late.

Mientras, en la sección "Quiénes Somos" se presenta el perfil de Diego Cazar, como editor; César Acuña, webmaster; Xavier Reyes, editor; Yalilé Loaiza, editora multimedia; Gabriela Ruiz, cronista; Wilo Ayllón, ilustrador; Juan Francisco Trujillo, redactor; Diana Romerio, cronista y editora. También consta el colectivo de fotorreporteros Fluxusfoto, que realizan trabajos documentales audiovisuales y fotoperiodismo. En sus minibiografías o perfiles de presentación, siete de los ocho integrantes se califica como periodista, cronista o editor "de a pie". Son personas con formación en periodismo y comunicación, tanto en pregrado como en posgrado (seis de ocho), pero sobre todo con experiencia previa en medios tradicionales como revistas y periódicos (cinco de ocho).

Los emprendimientos también surgen como una respuesta a varias necesidades sociales, necesidades que colocan al ciudadano común como protagonista en la selección de la información que va a consumir y, en el caso de La Barra Espaciadora, a los periodistas como protagonistas de su propio proceso editorial. Es algo que caracteriza a muchos em- 
prendimientos en América Latina (Knight Center Journalism in the Americas 2017), el hecho de arrancar procesos digitales innovadores, pero con todo el bagaje que muchos profesionales han adquirido en medios tradicionales y con los nuevos aprendizajes que han ido incorporando en sus rutinas de trabajo, como efecto de la era digital.

En este empeño, uno de los aspectos que resalta tanto en la página web como en la entrevista con el representante del medio, son las iniciativas de financiamiento. Mientras la historia de los medios tradicionales, como los periódicos, por ejemplo, revela una práctica rentable vía suscripciones, la opción no es precisamente la más rentable en el caso de los nuevos modelos de negocio que suponen los medios digitales. En el mundo se habla de micropagos, que consisten en pagos reducidos para acceder a contenidos específicos; los denominados pay per view, pagos por consumo; membresías, y la tradicional publicidad insertada (Dosdoce.com 2014). Como complemento a estas opciones, surgen otras modalidades como el crowfunding, un modelo empleado en medios on line, basado en la financiación de un proyecto por parte de varias personas. En España, este tipo de financiamiento se presentó como una opción ya en 2014 para proyectos de instituciones artísticas e incluso de la industria musical y del cine. Un estudio realizado en el mismo año revela que esta también fue una opción para proyectos pioneros en Argentina y Brasil, donde el crowfunding se presentó como una opción aunque no necesariamente como un método efectivo de financiamiento (Rencoret 2015).

En el caso de La Barra Espaciadora, el proyecto recurre a dos fórmulas de financiamiento. La primera, la fórmula tradicional, a través de segmentos para contenido comercial (publirreportajes), además de espacios publicitarios para banners de anunciantes. Por ejemplo, actualmente el portal tiene como anunciantes a libretas y cuadernos de la marca Betero, a productos de serigrafía de la marca Urku y un producto desconocido, denominado Tripa Mistic, descrito como "novela distópica", pero cuya página aún se encuentra en construcción.

La segunda fórmula es la creación de una red de patrocinadores del portal - que bien podría encajar en la definición del crowfunding-, que se promociona en el portal como la 'Red de Madrinos y Padrinas de La Barra' y que consiste en una invitación a "asiduos lectores, colaboradores del proyecto, amigos y diferentes profesionales y todos quienes creen en nuestros productos, en nuestro trabajo", para que contribuyan con aportes económicos permanentes que permitan sostener el proyecto. Se trata de paquetes mensuales, trimestrales, semestrales y anuales, con cuotas desde USD 15, hasta USD 150, que en su primer año de ejecución (2018) se proponía cubrir los gastos de los colaborares de contenido.

En esta misma línea, se podría incluir a una tercera vía de financiamiento que, sin embargo, no es considerada relevante por ser eventual. Esta es la tienda de artículos de LBE, que, al desplegarse, ofrece dos ediciones de libros compilatorios de textos publicados en LBE, libretas de apuntes de uno de sus auspiciantes y camisetas con publicidad del medio. 
A continuación, presentamos de manera gráfica los principales cambios e innovaciones identificados en el proyecto de periodismo narrativo La Barra Espaciadora, los cuales revelan una disposición por innovar, por adaptarse a nuevas dinámicas de trabajo.

\section{Tabla 1}

\section{Principales cambios en innovaciones}

\section{La Barra Espaciadora}

\begin{tabular}{|c|c|c|}
\hline Apropiación tecnológica & Dinámicas de trabajo & Iniciativas de sostenibilidad \\
\hline $\begin{array}{l}\text { Incorporación de un canal } \\
\text { de YouTube, para identificar } \\
\text { al medio y para difundir } \\
\text { contenido. }\end{array}$ & $\begin{array}{l}\text { Incorporación de elementos } \\
\text { visuales en las narrativas: uso de } \\
\text { formatos con mayor cantidad de } \\
\text { fotografías e incluso con videos. }\end{array}$ & $\begin{array}{l}\text { Fórmula tradicional: } \\
\text { espacio para anunciantes y } \\
\text { publirreportajes. }\end{array}$ \\
\hline $\begin{array}{l}\text { Incorporación de audios } \\
\text { y videos en la publicación } \\
\text { de algunos trabajos } \\
\text { periodísticos. }\end{array}$ & $\begin{array}{l}\text { Inclusión de prácticas de trabajo } \\
\text { colaborativo, con periodistas de } \\
\text { otros medios. }\end{array}$ & $\begin{array}{l}\text { Fórmula innovadora: una red } \\
\text { de patrocinadores o aportantes } \\
\text { permanentes. }\end{array}$ \\
\hline $\begin{array}{l}\text { Capacidad para influir en la } \\
\text { agenda mediática y la agenda } \\
\text { pública, a través del uso de } \\
\text { plataformas digitales (canal } \\
\text { YouTube); un ejemplo de } \\
\text { ello es la investigación del } \\
\text { caso Furukawa. }\end{array}$ & $\begin{array}{l}\text { Apuesta por contenidos } \\
\text { editoriales propios: cinco } \\
\text { secciones temáticas: cultura, } \\
\text { libertades, derechos, planeta y } \\
\text { frontera cautiva; un apartado } \\
\text { para reportajes más amplios e } \\
\text { informes 'dosieres'; además de la } \\
\text { recuperación de los principios de } \\
\text { la profesión. }\end{array}$ & $\begin{array}{l}\text { Una tercera opción, aunque no } \\
\text { permanente: una tienda virtual } \\
\text { con productos editoriales y } \\
\text { publicitarios del medio. }\end{array}$ \\
\hline
\end{tabular}

\footnotetext{
Intento por incorporar en contenidos periodísticos, elementos propios del lenguaje en internet, como la multimedialidad, la hipertextualidad y la interactividad, aunque esta última en menor medida.
}

Fuente: entrevista con representante de La Barra Espaciadora y observación de página web del medio. Elaboración: Paulina Escobar

\subsection{La Comunicación estratégica aplicada desde la cotidianidad. Caso Orión}

La agencia Orión Comunicación Estratégica es una empresa con base en Quito, que lanzó el proyecto 2017, aunque su fundadora tiene una trayectoria en este campo profesio- 
nal de más de 20 años. En la actualidad su trabajo se enfoca en la comunicación estratégica y comunicación política, con especial énfasis en las pequeñas y medianas industrias (pymes).

Si se concibe el concepto de hipermediación como la llegada de la tecnología a la vida profesional cotidiana y que por esta se han cambiado los límites que separan lo interior de lo exterior, para algunos profesionales de la comunicación esta ruptura ha hecho que la aplicación del mencionado concepto permita expandir los límites tradicionales de trabajo con los clientes; esto se ha dado porque los artículos smart como el computador o el celular, tienen una fácil manipulación y además en algunas ocasiones de manera simultánea, aunque no en el mismo lugar físico, se da una respuesta inmediata a las demandas de nuestros clientes.

Pero también es importante comprender que así como se solucionan problemas, también se generan otros, porque hablamos de una interacción de un flujo de comunicación que es bidireccional tanto de adentro para afuera como de afuera para adentro. En nuestro caso de análisis, esta disyuntiva es evidente en la relación con los clientes. Esto, debido a que una hipermediación también puede ser una trama de procesos de intercambio, producción y consumo simbólico que engloba una gran cantidad de sujetos, medios y lenguajes interconectados tecnológicamente.

Para hablar de interfaces como una cara visible de las hipermediaciones, para un comunicador que prácticamente ha crecido con el desarrollo tecnológico ocurrido en los últimos 20 años, este proceso de adaptación es cada vez más complejo porque siempre hay cosas nuevas por aprender, como el caso de la utilidad y potencialidad de los programas de edición, diseño, manejo de diversas plataformas o artículos tecnológicos y los mismos equipos, como las grabadoras de antaño que antes eran análogas y ahora son digitales. Es el caso de la experiencia de la agencia Orión, cuya creadora ha vivido el proceso de transformaciones y adaptaciones desde el inicio de su carrera profesional como periodista y luego como comunicadora hasta transformarse en la emprendedora de su propio proyecto de comunicación.

Si el nuevo mapa del mundo de la comunicación está dominado por dos ideologías hegemónicas: la vía del discurso único de la economía y la el impacto de las tecnologías con el desasosiego que implica el uso de las redes sociales, también es cierto que hoy encontramos cierta resistencia al cambio, lo cual ocurre incluso en aquellos profesionales de la comunicación que trabajan con intangibles como la reputación. Asimismo, en el caso del manejo de la identidad, aun es difícil conjugar la imagen real con la del mundo digital, algo que, en otros campos profesional, sería indivisible. Podríamos afirmar incluso que el hecho de preservar el tradicional "buen nombre a través del boca a boca" es algo que debería irse combinando de manera paulatina con la reputación digital, donde "el boca a boca" vendría a ser la multiplicación de nuestros seguidores y de nuestras propias redes sociales.

A pesar de que, en la actualidad, la comunicación digital es una herramienta que se utiliza como prioridad, todavía tenemos ejemplos como el de Orión, en que la mayoría de clientes son contactados por una vía directa o por clientes que estuvieron satisfechos por su buen trabajo y los refirieron. Otros, en cambio, son producto de la interacción que tuvieron 
cuando la gerente trabajaba en medios de comunicación. Por ejemplo, dentro de nuestro estudio vemos que aunque la agencia no cumple con todos los pasos para completar un proceso estratégico de apropiación e integración de redes en sus estrategias de comunicación, actualmente es un emprendimiento con proyección de crecimiento en el área de la comunicación estratégica.

Tabla 2

\begin{tabular}{ll}
\multicolumn{1}{c}{$\begin{array}{c}\text { Pasos para un proceso estratégico del apropiación e integración } \\
\text { de redes en estrategias de comunicación } \\
\text { Agencia Orión* }\end{array}$} \\
\hline Pasos & $\begin{array}{l}\text { Nivel de } \\
\text { cumplimiento }\end{array}$ \\
\hline $\begin{array}{l}\text { Análisis de las necesidades de los clientes e identificación de los } \\
\text { públicos o clientes. }\end{array}$ & No \\
\hline $\begin{array}{l}\text { Análisis de las necesidades de los clientes e identificación de sus } \\
\text { objetivos }\end{array}$ & No \\
\hline $\begin{array}{l}\text { Ha escogido la herramienta adecuada y la creación de perfiles de } \\
\text { sus clientes }\end{array}$ & $\mathrm{Si}$ \\
\hline $\begin{array}{l}\text { Planifica contenidos para cada medio así como su línea editorial } \\
\text { y normas de uso. }\end{array}$ & $\mathrm{Si}$ \\
\hline Definición de la gestión del administrador de medios o redes & No \\
\hline Crea comunidades de interés & $\mathrm{Si}$ \\
\hline Evalúa todos los pasos de la estrategia & No \\
\hline
\end{tabular}

\section{Leyenda:}

Sí: a pesar de que el inicio del emprendimiento fue orgánico, en la actualidad existe una planificación en el manejo de la imagen que es utilizada en redes.

No: Orión trabaja con sus clientes, quienes son referidos únicamente de manera personal; por tal motivo la observación del entorno de redes es una tarea que ha quedado desplazada a un segundo plano.

Fuente: entrevista con representante de la agencia Orión.

Elaboración: Liza Bahamonde

\section{Discusión y conclusiones}

Los emprendimientos periodísticos en la era digital no solo evidencian una apuesta editorial, evidencian también una postura ante la hegemonía de las propuestas editoriales de los medios convencionales. Representan una posibilidad de multiplicar las voces de los protagonistas de los proyectos, así como de las fuentes de información. Con respecto a las 
audiencias, también otorgan un papel preponderante a los ciudadanos, frente a la que estos tienen en la agenda mediática convencional.

La posibilidad de emprender un proyecto periodístico es además una puerta al aprendizaje y a la constante innovación y actualización de conocimientos, así como de apropiación tecnológica de la cultura digital. Los dispositivos y herramientas que provee la tecnología constituyen canales que permiten consolidar propuestas editoriales desde su implementación y a lo largo del mismo proceso.

Mientras en el mundo de los medios tradicionales, que incluso han trasladado sus versiones a plataformas digitales, el financiamiento que permite sostener los proyectos está ligado a la estructura y prácticas propias de estos medios, en los medios digitales, el financiamiento es quizá una de las mayores dificultades, porque de este aspecto depende la rentabilidad y sostenibilidad de los proyectos. Aun cuando en el mundo existen ya modelos de negocio basados en el crowfunding, en países como en el nuestro son prácticas no tan expandidas, quizá por los comportamientos de las audiencias. Eso no implica que ciertos proyectos ya hayan emprendido iniciativas para financiarse con el aporte de redes de aportantes.

La realidad en Ecuador revela que existe espacio para la puesta en escena de proyectos innovadores, que no solo incorporen tecnología, como canales de YouTube, contenidos con realidad virtual o coberturas mediante sistemas de live streaming; sin embargo, la principal apuesta es el manejo de nuevas narrativas digitales, donde la calidad del contenido y el estricto apego a los fundamentos del periodismo, como la ética, verificación de información, contextualización y la apertura a multiplicidad de voces seguirá siendo lo más importante al momento de ejercer el periodismo.

La comunicación en los últimos años ha experimentado un profundo cambio y entender a la interfaz es algo vital para quienes trabajan en lo digital. Esto ha sucedido con los dispositivos y programas que se utilizaban hace no más de 20 años y la cascada de información a la que estamos expuestos en la actualidad. Esto genera una serie dificultades al momento en que las personas se actualizan, porque, aunque se encuentren en un continuo proceso de aprendizaje y redescubrimiento, siempre llega (y llegará) una tecnología innovadora que remplace la precedente antes de que podamos asimilar como protagonistas de este proceso la utilidad de los recursos digitales.

Muchos piensan que el comunicador, por estar cerca de las diferentes plataformas tecnológicas, necesariamente debe estar familiarizado con estos nuevos procesos de comunicación que se producen en la red. Se trata de una falsa percepción, ya que genera a una expectativa equivocada, porque el comunicador no es un erudito en el manejo de la comunicación en la red, a pesar de que conviva con ella a diario.

Este es un tiempo de autoaprendizaje especialmente para las generaciones anteriores a los denominados "milenials" puesto que muchos profesionales de la comunicación no ven el verdadero potencial de la comunicación digital y cómo esta puede acortar tiempo y espacio en nuestro trabajo. Consideramos que es una tarea inconclusa y que los profesionales de 
la comunicación tenemos la obligación de actualizarnos constantemente.

El discurso de la economía de la información ha permitido entender el valor que puede tener una marca o reputación, mucho más en el contexto de la era digital en que todo se multiplica o masifica, generando un verdadero valor comercial y monetario. Quienes no consideren la importancia de estos aspectos, de una manera sostenible, son susceptibles de, en poco tiempo, quedar expuestos al olvido digital.

\section{Referencias}

Corporación Andina de Fomento (CAF). 2013. Emprendimientos en América Latina. Bogotá: CAF.

Centro Knight para Periodismo en las Américas. 2018. Innovadores en el periodismo latinoamericano. Austin: Open Society Foundations.

Charest, Francine, Anne-Marie Gauthier y François Grenon. 2013. “Appropiation et stratégies d'intégration des médias sociaux par les professionnels de la communication”. Communication et organisation (43): 269-80.

Centro Internacional de Estudios Superiores de Comunicación para América Latina (Ciespal). 2012. Mapa de medios digitales del Ecuador. Quito: Ciespal.

Coffey, Amanda y Paul Atkinson. 2003. Encontrar el sentido a los datos cualitativos: Estrategias complementarias de investigación. Medellín: Universidad de Antioquia.

Costa, Joan. 2015. El paradigma DirCom. Barcelona: Costa Punto Com.

Dosdoce.com. 2014. Nuevos modelos de negocio en la era digital. Madrid: Centro Español de Derechos Reprográficos.

Escobar, Paulina. 2018. "Comment l'usage de Twitter a changé les pratiques journalistiques des journalistes du journal équatorien El Comercio". Communication 35 (2). http://doi.org/10.4000/ communication. 8660

Espae-Espol. 2018. Global Entrepreneurship Monitor Ecuador 2017. Guayaquil: Espae-Espol.

Fernández, Katiuska, Alma Vallejo y Lewis McAnally. 2015. “Apropiación tecnológica: una visión desde los modelos y las teorías que la explican”. Perspectiva Educacional 54 (2): 109-25.

Fundación Friedrich-Ebert-Stiftung. 2015. Comunicación en mutación. Bogotá: Fundación FriedrichEbert-Stiftung FES Comunicación.

Garrido, Francisco. 2004. Comunicación estratégica. Madrid: Gestión.

Gómez Cruz, Edgar. 2007. Metáforas de Internet. Barcelona: UOCPress.

Gutiérrez, Elena y María Teresa LePorte (eds.). 2013. Tendencias de la comunicación de instituciones. Barcelona: UOCPress. 
Knight Center Journalism in the Americas. 2017. Periodismo innovador en América Latina. Austin: Knight Center Journalisme in the Americas.

Mercier, Arnaud y Nathalie Pignard-Cheynel. 2014. "Mutations du journalisme à lère du numérique: un état des travaux. Revue francaise des sciences de l'information et de la communication". Revue Française des Sciences de l'information et de la comunication 5 (3). https://doi.org/10.4000/ rfsic.1097.

Montalvo-Castro, Jorge. 2016. “Emprendimientos digitales y diseño de modelos de negocio: investigación aplicada en estudiantes de la Carrera de Comunicación”. Revista Contratexto 25. https://revistas. ulima.edu.pe/index.php/contratexto/issue/view/61

Pérez-Latre, Francisco Javier. Y Alfredo Sánchez-Tabernero. 2012. Innovación en los medios: la ruta del cambio. Pamplona: EUNSA.

Quezada, Cristóbal y Martín Pérez. 2016. “De la telegrafía sin hijos a radiodifusión: apropiación tecnológica de la radio en Chile, 1901-1931”. Revista de Historia Iberoamericana 9 (1): 103-25.

Real Academia Española (RAE). 2019a. “Emprender”. Diccionario de la Lengua Española. https://dle. rae.es/?id=Esip2Nv

. 2019b. "Sociedad". Diccionario de la Lengua Española. https://dle.rae.es/sociedad?m=form

Rencoret, Nathalie. 2015. "Crowdfunding como iniciativa al periodismo, estudio de casos en los países de Argentina y Brasil”. Tesis de grado. Universidad Técnica Particular de Loja.

Rivera, Diana y Dominique Rosero. 2017. “Análisis de las prácticas y técnicas periodísticas en el periodismo ecuatoriano. Caos de estudio: Ecuavisa, El Comercio y Ecuador en Vivo”. Razón y Palabra 21 (2-97): 475-85.

Scolari, C. 2013. Hipermediaciones: Elementos para una Teoría de la Comunicación Digital Interactiva. Quito: Gedisa.

Top Seeds Lab. 2015. Dossier de prensa. Madrid: Top Seed Labs. 